\title{
Aplicación de la regeneración ósea guiada con injerto en bloque de mentón en la colocación de implantes
}

\author{
Application of guided bone regeneration by placing chin block graft in \\ the placement of implants
}

\section{Resumen}

Los defectos óseos alveolares que se originan por la pérdida de una pieza dentaria pueden dificultar diferentes procedimientos odontológicos, como la colocación de implantes. Ante esta situación, se pueden desarrollar técnicas que permitan aumentar el reborde, sea este horizontal o vertical. La regeneración ósea guiada(ROG) es utilizada desde los años 90 del siglo pasado, basándose en los conceptos de la regeneración tisular guiada, utilizando una membrana que puede generar una mejor condición para la formación de hueso. En el presente caso clínico se desarrolla el diagnóstico, plan de tratamiento, técnica quirúrgica, así como las indicaciones postoperatorias para la mejora en la recuperación del paciente.

Palabras clave: Defecto óseo alveolar, injerto en bloque de mentón, regeneración ósea guiada, implante dental.

\section{Abstract}

Alveolar bone defects that result from the loss of a tooth, can hinder different dental procedures such as implants. In this situation, you can develop techniques to increase the alveolar ridge, either horizontally or vertically. Guided bone regeneration (GBR) is used since the 90s, based on the concepts of guided tissue regeneration, using a membrane that can generate better conditions for bone formation. In this case the clinical diagnosis, treatment planning, surgical technique and post-operative indications for improvement in the patient's recovery, are developed.

Keywords: Alveolar bone defect; chin block graft; guided bone regeneration; dental implant
Caso Clínico

\author{
Marco Rivas Sarmiento ${ }^{1}$, \\ Donald Ramos-Perfecto².
}

1. Unidad de Posgrado de la Facultad de Odontología de la Universidad Nacional Mayor de San Marcos. Segunda Especialidad en Periodoncia, Perú.

2. Departamento Académico de Ciencias Básicas de la Facultad de Odontología de la Universidad Nacional Mayor de San Marcos, Perú.

Correspondencia

C.D. Marco Rivas Sarmiento

Camino Real del Inca 310, Lima 32, Perú

Correo electrónico: marsa12@hotmail.com

Coautor:

Ramos: dramos_37@hotmail.com

Aprobado: 30-10-15

\section{Introducción}

Los defectos óseos alveolares generados por la extracción dental pueden generar, en el trascurso de un ańo, una pérdida aproximada de entre el 25 y $50 \%$ del ancho óseo y dos terceras partes de la pérdida se dan en los tres primeros meses $^{1,2}$. Diferentes técnicas son utilizadas para el incremento del reborde óseo, como la regeneración ósea guiada (ROG), injerto por oposición, injerto por interposición, expansión ósea, distracción osteogénica y revascularización de colgajos $^{3,4}$, siendo el gold standard en la regeneración ósea, el uso de injertos autógenos en la cirugía reconstructiva de maxilares ${ }^{5,6,7}$.

La zona donante para el aumento óseo de maxilares pueden ser extraorales, como hueso de calota, cresta iliaca ${ }^{8}$, costilla y tibia, los cuales no son muy recomendados por su morbilidad, riesgos asociados y la necesidad de hospitalización. De las zonas intraorales se puede obtener hueso del cigomático, rama mandibular, tuberosidad de maxilar y sínfisis mandibular, que son los más usados por su menor riesgo asociado, mejor posoperatorio y pudiéndose realizar en el consultorio ${ }^{7}$.

La sínfisis o mentón mandibular presenta en su zona vestibular la inserción del músculo borla de la barba, entre el fondo del surco y el borde inferior de la mandíbula, nace a la altura del relieve óseo de la raíz del incisivo lateral y en la cara profunda de la mucosa del surco vestibular. Esta zona vestibular es inervado por el nervio incisivo y algunas ramas del mentoniano, siendo suficiente la aplicación de anestesia infiltrativa en la zona para lograr el bloqueo del nervio, ya que este tiene un recorrido por fuera del hueso ${ }^{9}$

El mentón es un hueso de fácil acceso, presentando propiedades biológicas como, que la cortical del injerto sirve como una membrana osteoconductora y la porción medular le da características osteoinductoras ${ }^{7}$, osteogeneradora y por ser un injerto corticomembranoso se revasculariza más rápido que los injertos de gran grosor ${ }^{10}$, siendo su reabsorción más lenta en comparación con otros injertos autógenos, por lo que favorecería la regeneración a largo tiempo. Así también presenta una mayor concentración de proteínas morfogenéticas óseas que permiten una mayor regeneración ósea ${ }^{11,12,13}$. Las dimensiones máximas de injerto de mentón que se pueden obtener es de $35 \mathrm{~mm}$ de largo, $4 \mathrm{~mm}$ de ancho y $10 \mathrm{~mm}$ de altura, aproximadamente para todas las dimensiones ${ }^{14}$, aunque va a depender de la calidad ósea que presente el paciente. Como regla se puede tomar injertos de mentón siguiendo la regla del 5, es decir $5 \mathrm{~mm}$ lejos del ápice radicular, 5 del borde inferior del mentón y 5 del nervio mentoniano ${ }^{1}$. Los estudios han demostrado que los injertos en bloque o corticomembranoso tienen una alta tasa de supervivencia, por encima del $90 \%$, injertos que han incrementado rebordes para colocar implantes ${ }^{12,15,16}$. 
La ROG es una técnica que se conoce a inicios de los ańos 90 del siglo XX, en un inicio su aplicación se da para incrementar mandíbulas atróficas y hoy en día es muy usada para incrementar rebordes donde hay que colocar implantes. Esta técnica se basa en los principios creados para la regeneración tisular guiada, en la cual se coloca una membrana de barrera para proteger los defectos óseos de la invasión de tejido blando, para que se forme hueso, hueso que se forma de células precursoras de osteoblastos que están presentes en los espacios medulares, endostio y periostio. Estos están localizados frecuentemente en las inmediaciones de vasos sanguíneos próximos a la superficie ósea y son capaces de responder a la inducción osteogénica. Los osteoblastos no migran ni se dividen, por lo que dependen de células progenitoras mesenquimatosas localizadas en la superficie ósea adyacente para su formación y posterior formación de hueso ${ }^{3,17,18}$.

La presentación del caso clínico tiene por objetivo dar a conocer una técnica segura, predecible en el manejo del incremento óseo ante rebordes residuales muy estrechos.

\section{Reporte de caso}

Paciente de 52 años, proveniente de Lima, siendo su motivo de atención el colocarse un implante dental en reemplazo de la pieza dentaria 22 extraída. Al desarrollar la historia clínica médica, no indica padecer alguna enfermedad sistémica, descartando algún factor de riesgo asociado a ello. Al examen clínico estomatológico, se presenta asintomático, identificando un puente fijo en bandera a nivel de 22 y 23, observándose una depresión a nivel de la mucosa del póntico 22 (fig. 1). El estudio topográfico de la depresión evidencia volumen óseo reducido (fig. 2). La paciente presenta exposición de las raíces a nivel del tercio cervical, así como una pérdida parcial de papila en el sector antero inferior de la mandíbula, e insuficiente volumen de reborde en sentido vestí- bulo palatino para la colocación de un implante.

\section{Diagnóstico}

1. Defecto óseo de reborde clase I según Seibert a nivel de póntico 22

2. Edéntula parcial superior Clase III de Kennedy

3. Recesión gingival Miller clase III Pzas. 31, 32, 41, 42.

\section{Plan de tratamiento}

Identificados los defectos, el plan de tratamiento va dirigido a incrementar el reborde óseo a nivel del póntico 22, con la finalidad de colocar en dicha zona incrementada un implante. Por lo que se realiza un ROG con injerto en bloque de mentón, relleno óseo particulado y membrana reabsorbible de colágeno, luego de un periodo de consolidación del injerto de 6 meses se realizará la colocación del implante.

\section{Técnica quirúrgica}

\section{Zona receptora}

Se coloca lidocaína al $2 \%$ por infiltración, en la zona receptora de 11 a 23 , luego realizamos una incisión supracrestal y 2 incisiones liberantes con hoja de bisturí $\mathrm{N}^{\circ} 15$, se decola a espesor total para exponer el defecto (fig. 3).

\section{Zona donante}

Previo a la anestesia infiltrativa se realiza una incisión a espesor parcial con inclinación de $45^{\circ}$ a $5 \mathrm{~mm}$ de distancia de los ápices de los incisivos inferiores y se decola la mucosa. Se realiza una segunda incisión a espesor total con inclinación de $90^{\circ}$ y se decola hasta exponer la zona donadora (fig. 4). Tomando en cuenta la dimensión del defecto, se realizó una perforación circular con una trefina de $8 \mathrm{~mm}$ de diámetro y con una profundidad de $4 \mathrm{~mm}$ (fig. 5), se coloca un tornillo a nivel central del hueso perforado y mediante luxación se retira el bloque óseo, para ser remodelado y adaptado a la forma del defecto de la zona receptora (fig. 6).

\section{Enlace zona donante a zona receptora}

Una vez obtenido el injerto en bloque se procede a la decorticalización de la zona receptora hasta llegar a tejido esponjoso, para tener mayor irrigación del injerto (fig. 7). Se coloca el injerto fijándolo con el tornillo adaptado de $1,8 \mathrm{~mm}$ X $10 \mathrm{~mm}$ (fig. 8), se coloca hueso bovino como material de relleno en los espacios vacíos entre zona receptora e injerto (fig. 9). Se adapta una membrana de colágeno reabsorbible al lecho para cubrir el injerto (fig. 10). Se realizó la síntesis de los tejidos blandos en la zona receptora liberando el periostio en la base del colgajo para permitir el cierre libre de tensión y se sutura con ácido poliglicólico 5/0, mientras que la zona donadora se sutura en dos planos

\section{Indicaciones post-operatorias}

Se medica al paciente con clindamicina de $300 \mathrm{mg}$. vía oral cada 8 horas por 7 días. Dexametasona de $4 \mathrm{mg}$ por vía intramuscular por 2 días. Naproxeno sódico de $550 \mathrm{mg}$. vía oral cada 12 horas por 5 días, esto condicionado al dolor. Se le debe indicar que se enjuague la boca con clorexidina al $0.12 \% 2$ veces por día, por 10 días, así como colocarse hielo por fuera de la zona operada por 2 horas. Su dieta debe ser blanda complementándose con una formula tipo "vida Max" 1, su higiene debe ser continua y muy delicada en las zonas operada, por lo que se le recomienda usar cepillos dentales ultrablandos, así también se le indica un reposo de 3 días.

Se realizan controles al paciente a los 3 , 7 y 15 días, se le retira los puntos (fig. 11), a los 6 meses después se toma una nueva tomografía (fig. 12) y se observa que el ancho del hueso residual mide ahora $8 \mathrm{~mm}$ en la zona del injerto en bloque. Se realiza un colgajo a espesor total para retirar el tornillo (fig. 13) y se procede a la preparación del lecho óseo para colocar el implante cónico de 3,5 X 11.5 mm (fig. 14 y 15) se sutura el colgajo con ácido poliglícolico 5/0 y se toma una radiografía de control (fig. 16). Se indica al paciente los mismos cuidados posoperatorios que en la cirugía anterior. 


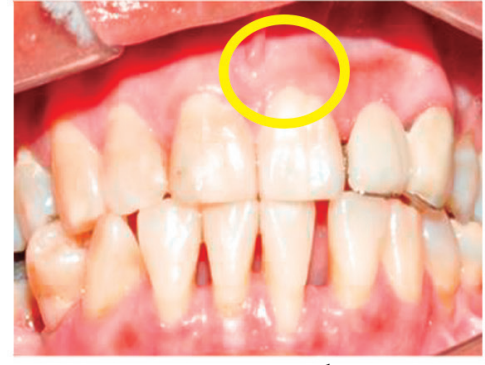

Fig.1. Vista intraoral previa

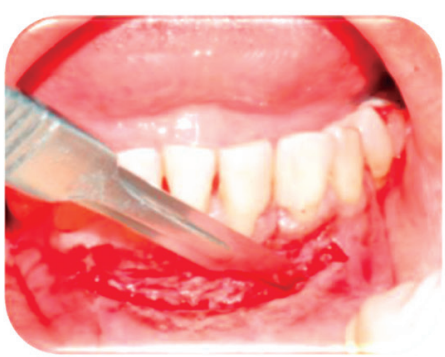

Fig. 4 Incisión a espesor parcial zona dadora

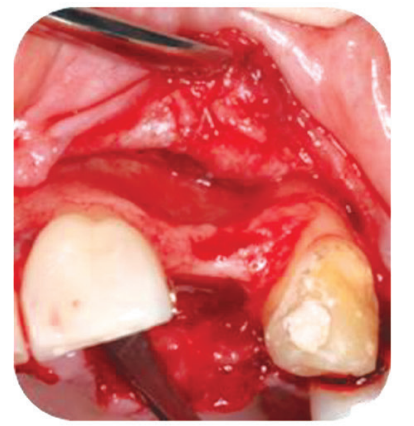

Fig. 7 Decorticalización de zona receptora

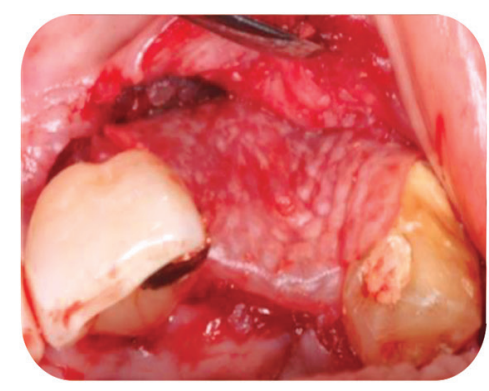

Fig. 10 Adaptación de membrana

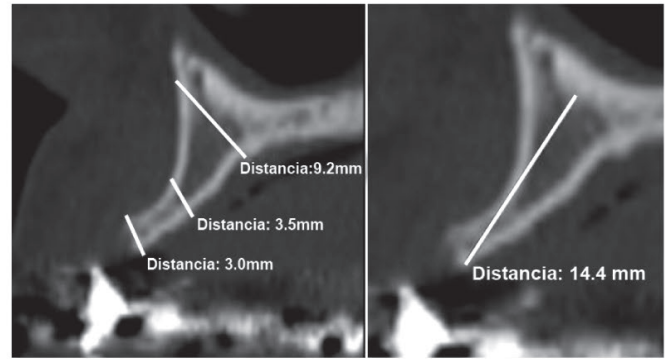

Fig. 2 La tomografía muestra las dimensiones del hueso

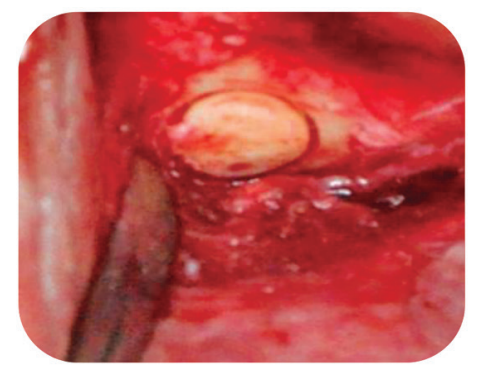

Fig. 5 Corte circular con trefina

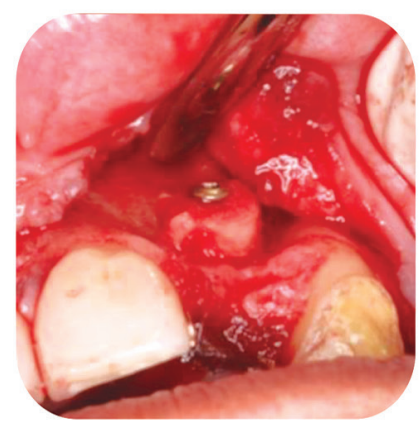

Fig. 8 Fijacion de injerto con tornillo

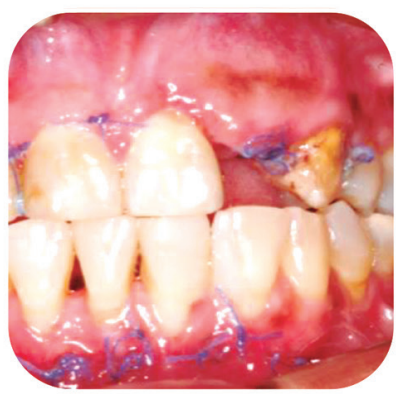

Fig. 11 Apréciese el aumento de volumen

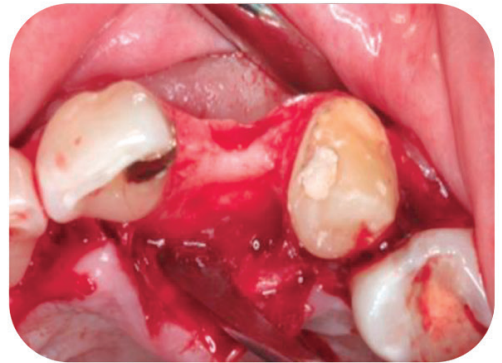

Fig. 3 Colgajo de zona receptora

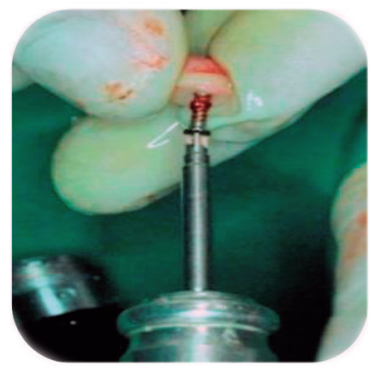

Fig. 6 Tornillo de figacion en bloque óseo

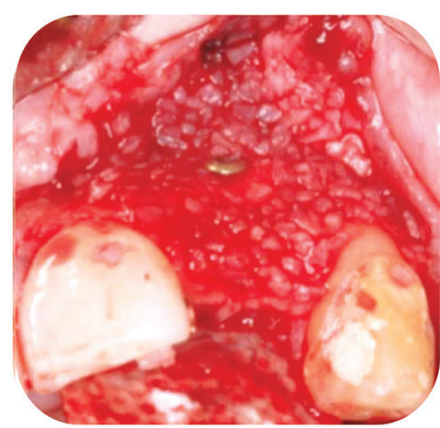

Fig. 9 Colocación de hueso particulado

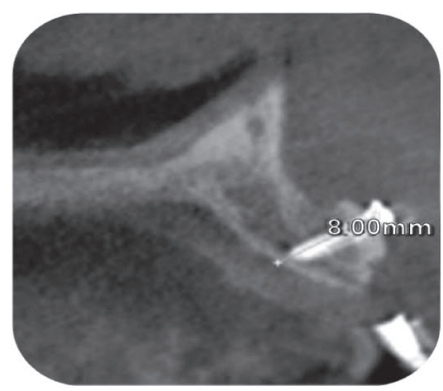

Fig. 12 Tomografía a los 6 meses obsérvese el incremento de volumen hueso

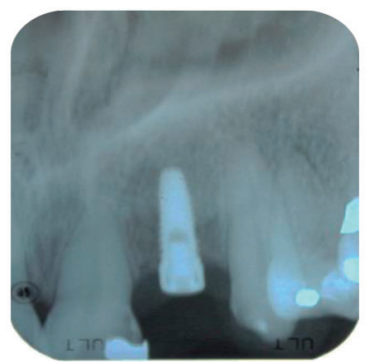

Fig. 15

Colocación de implante

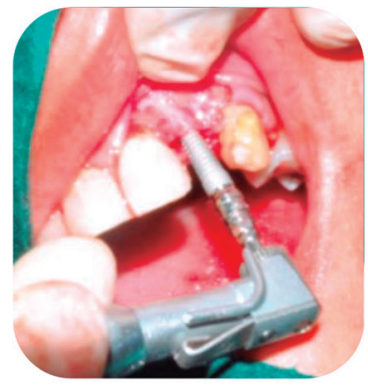

Fig. 16 Rx. permite ver el implante en el lecho óseo con ROG con injerto de mentón
Injerto consolidado
Preparación del lecho óseo 


\section{Discusión}

La ROG es una técnica predecible, que usa injertos corticomedulares autólogos en bloque, que tienen capacidad osteogénica, osteoinductiva y osteoconductora, así como su buena incorporación en el lecho receptor y su poca pérdida de volumen hacen que este tipo de injerto sea considerado como el injerto gold standard para el incremento de rebordes atróficos que van a recibir implantes. Similares conclusiones llegan a sustentar autores como Ávila Souza $^{19}$, Rachana ${ }^{20}$, Toscano ${ }^{1}$. Sobre la poca pérdida de volumen del injerto en bloque, Clementine ${ }^{15}$, en una revisión sistemática sobre injertos en bloque que incrementan rebordes para colocar implantes, manifiesta que la técnica de ROG es predecible en la recuperación de rebordes atróficos, con pérdidas por resorción del injerto de menos del $10 \%$ al colocar el implante.

Cardoso $^{6}$ realiza procedimientos para mejorar rebordes atróficos con injertos en bloque, para posterior colocación de implantes, observando que la media de aumento lateral obtenida en el momento de colocar el injerto óseo era de 6,5 $+/-0,33 \mathrm{~mm}$, que se reduce durante la cicatrización debido a la resorción del injerto en una media de $5.0+/-0,23$ $\mathrm{mm}$. La media de aumento vertical era de 3,4 +/- 0,66 $\mathrm{mm}$ en el injerto óseo y $2,2+/-0,66 \mathrm{~mm}$ en la colocación de implantes. La media de aumento lateral y vertical disminuyó en un 23,5 \% y 42 $\%$ respectivamente durante la curación del injerto óseo. En relación con la ganancia ósea, Chiapasco ${ }^{16}$ obtuvo resultados similares al ejecutar la técnica de ROG con membrana no reabsorbible e injerto en bloque luego de 6 a 8 meses logra colocar implantes en las áreas regeneradas con una media de ganancia de hueso de $4 \mathrm{~mm}$. En nuestro caso clínico, el aumento lateral fue de $5 \mathrm{~mm}$, resultados parecidos a los de $\operatorname{Cardoso}^{2}$ y un poco más que Chiapasco ${ }^{16}$.

En relación con las posibles complicaciones de esta técnica, se menciona que podría ser la falta de fijación del injerto a la zona receptora, lo cual no podría generar una buena cicatrización y por tanto no hay una buena consolidación del injerto en la zona receptora. Racha$\mathrm{na}^{20}$ indica el íntimo contacto del injerto en bloque con la zona receptora, su estabilidad en la zona es crucial para el éxito del procedimiento, por lo que indica que el tornillo de fijación esté bien colocado e insertado. Chiapasco ${ }^{4}$ en su revisión sobre los diversos procedimientos que aumentan rebordes alveolares deficientes para colocar implantes y rehabilitarlos, menciona que la causa más frecuente de complicaciones en los injertos en bloque es consecuencia de las dehiscencias de la mucosa, con exposición del injerto al medio intraoral. Esto probablemente por desplazamiento del injerto o desprendimiento de los puntos de sutura.

En otro estudio, Cardoso ${ }^{21}$ evalúa el efecto del hueso bovino desproteinizado y de la membrana de colágeno en la cicatrización de los injertos en bloque de mandíbula, obteniendo resultados de la media de reabsorción del injerto entre el grupo experimental y el grupo control sin membrana y hueso, donde el grupo experimental tenía $0,25 \mathrm{~mm}$ de resorción y el grupo control de 0,89 $\mathrm{mm}$. Concluyendo que la adición de hueso bovino más membrana podrían generar un aumento lateral de $5 \mathrm{~mm}$. Rachana $^{20}$ en su estudio manifiesta que la membrana de colágeno y ácido hialuronico pueden promover la regeneración ósea, a través o por su actividad en osteoblastos, lo que sugiere que la membrana reabsorbible favorece la regeneración ósea y reparación. En nuestro caso clínico, se colocó hueso particulado y membrana para un mayor porcentaje de éxito del injerto en bloque.

\section{Conclusión}

La Regeneración ósea guiada con injerto en bloque de mentón es una técnica predecible, con un porcentaje de éxito de más del $90 \%$, baja morbilidad, que puede ser utilizada en los aumentos horizontales de rebordes atrofiados por pérdida dental y que en un periodo de cicatrización del injerto de 6 meses es posible colocar el implante dental.

\section{Referencias Bibliográficas}

1. Tosacano N, Shumaker N, Holtzclaw D. The art of block grafting a review of the surgical protocol for reconstruction for alveolar ridge deficiency. J Implant Adv Clin Dent. 2010;2(2):45-66.

2. Misch C. Protesis dental sobre implantes. 1ra ed. Madrid: Elsevier; 2006.p.5-6.

3. Seibert JS, Salama H. Alveolar rigde preservation and reconstruction. Periodontol 2000 1996;11:69-84.
4. Chiapasco M, Zaniboni M, Boisco M. Augmentation procedures for the rehabilitation of deficient edentulous ridges wiht oral implants. Clin. Oral Implant. Res. 2006;17(Suppl. 2):136-159.

5. Pikos Michael A. Mandibular block autografts for alveolar ridge augmentation.Atlas oral maxillofacial surgery. Clinic N Am 2005;13:91-107.

6. Cordaro L, Amade D, Cordaro M. Clinical results of alveolar ridge augmentation whit mandibular block bone grafts in partially edentulous patients prior to implant placement. Clin Oral Impl Res. 2002;13:103-111.

7. Smolka W, Eggensperger N, Carollo V, Ozdoba C, Lizuka T. Changes in the volume and density of calvarial split bone grafts after alveolar ridge augmentation. Clin Oral Impl Res 2006;17(2):149-55.

8. Pereira E, Messias A, Dias R, Judas F, Salvoni A, Guerra F. Horizontal resorption of fresh -frozen corticocancellous bone blocks in the reconstruction of the atrophic maxilla at 5 months. Clin oral impl Res. 2014; 25(Suppl 10):354.

9. Romanelli H. Fundamentos de cirugia periodontal. 1 ra ed. Colombia: Amolca; 2004.p.41-53.

10. Nevins, R. Melloning, J.T. The advantaje of localized ridge augmentation prior to implant placement: A two stage event. Int J Period Rest Dent 1994;14:97-111

11. Jimi E, Hirata S, Shin M, Yamazaki M, Fukushima H. Molecular mechanisms of BMP-induced bone formation: cross-talk between BMP and NF- $\mathrm{BB}$ signaling pathways in osteoblastogenesis. Japanese Dental Science Review. 2010;46(1):33-42.

12. Schwartz-Arad D, Levin L. Intraoral autogenous block onlay bone grafting for extensive reconstruction of atrophic maxillary alveolar ridges. J Periodontol 2005;76:636-641.

13. Ramos PD, Garcia DJ, Sueldo GL. Regeneración osea guiada con injerto autógeno de mentón. Odontol Sanmarquina 2007;10(2):33-36. 
14. Levin L, Nitzan D, Schwartz-Arad D. Succes of dental implants placed in intraoral block bone grafts. J Periodontol 2007;78:18-21

15. Clementini M, Morlupi A, Agrestini C, Ottria L. Success rate of dental implants insertd in autologous bone graft regenerated areas: A systematic review. Oral Implantol 2011;4(3-4):3-10.

16. Chiapasco M, Abati S, Romeo E, Vogel G. Clinical outcome of autogenous bone blocks or guided bone regeneration with e-PTFE membranes for the reconstruction of narrow edentulous ridges. Clin Oral Impl res 1999;10:278-288.

17. Novaes A. Cirugía periodontal con finalidad protésica. 1ra ed. Sao Paulo, Amolca;2001:119-123.

18. Nappe CE, Baltodano CE. Regeneracion osea guiada parael aumento vertical del reborde alveolar. Rev. Clin. Periodoncia Implantol. Rehabil. Oral. 2013;6(1):38-41.

19. Avila SF, Farnezi BAP, Marcondes AA, Ponzoni D, Bufulun LG, Jorge BFBD. Reconstruction of maxillary ridge atrophy caused by alveolar trauma, using autogenous block bone graft harvested from chin: a case report. J osseointegr 2014;6(2):21-27.

20. Rachana C, Sridhar, Rangan AV, Rajani V. Horizontal ridge augmentation using a combinatión approach. J indian soc periodontal 2012;16(3):446-450.

21. Cordaro L, Torsello F, Morcavallo $S$, di Torresanto VM Effect of bovine bone and collagen membranes on healing of mandibular bone blocks: a prospective randomized controlled study. Clin Oral Implants Res 2011;22(10):1145-50 